\title{
You are what you eat - myth or reality: influences of diet on the body
}

\author{
Evelyn Medawar ${ }^{1,2,3}$ \\ ${ }^{1}$ Max Planck Institute for Human Cognitive and Brain Sciences, Leipzig, Germany \\ ${ }^{2}$ Berlin School of Mind and Brain, Humboldt-Universität zu Berlin, Germany \\ ${ }^{3}$ Charité - Universitätsmedizin Berlin; Humboldt-Universität zu Berlin, Germany
}

\begin{abstract}
Dietary patterns and their influences on the body and in particular on behaviour are oftentimes a matter of debate in the public, but also in the scientific community. In a set of studies, including cross-sectional, epidemiological and interventional designs, we aim to address the relationship between diet and the body in a multitude of ways. We focus in particular on plant-based diets, i.e. restricting animal-based products. We found better weight status and personality differences in individuals restricting animal-based product intake. However, our results only represent a small puzzle piece to a field only starting to grow.
\end{abstract}

\section{Background}

Meat-restrictive eating patterns are debated to either convey health benefits or risks (reviewed in Medawar et al. 2019). Recent studies indicated conflicting evidence for emotional health, including higher depression and higher neuroticism in vegetarians (Hibbeln et al. 2018), in contrast to negative findings concerning this link (Medawar et al. 2020). Recently, it has been suggested that not meat-restriction per se, but the number of excluded food groups was associated with higher depressive scores (Matta et al. 2018), which was shown to be the inverse relation in the Leipzig based LIFE Adult study (Medawar et al. 2020; Loeffler et al. 2015).

The aim of my $\mathrm{PhD}$ project is to assess, whether plantbased diets, i.e. a high fibre diet, influence our decisions of what we would like to eat. We postulate that potential effects are mediated by bacteria found in our gut.

The project is divided into 4 sub-studies: in observational studies (study 1+2) we investigated the effect of a single meal choice on well-being and satiety in German student cafeterias. In a cross-sectional study (study 3) we investigate whether the frequency of consumption of animal-derived products is associated with weight status, emotional health and personality traits in the local LIFE Adult study, a population-based study with over 10,000 participants in Leipzig. In an intervention study (study 4) we examine, whether a two-week high-fibre dietary intervention affects food wanting, memory performance and their neural correlates as well as brain structure and microstructure. Further, we test whether biomarkers, such as microbial status, serum levels of proteins and hormones mediate the observed effects.
The nationwide single-meal study indicates, that meatbased meals mediate higher post-meal contentment on average, whereas plant-based meals are more frequently chosen when pre-meal mood is higher. The local crosssectional study shows that less animal-based foods links to better weight status, but not to depressive symptoms the latter may be better explained by differences in demographics and personality traits. Results of the gutbrain-axis intervention (study 4) will be available soon. Overall, long-term interventional trials are needed to test diet-induced bottom-up signaling from the gut to the brain and to clarify the underlying mechanisms, which may in particular involve the gut microbiome. The latter is a hotly debated candidate for unraveling the myth and moving closer to a real diet-body relationship.

\section{Acknowledgments}

Those go especially to all study participants, who were willing to contribute to basic research, to all study team members, and last but not least to my supervisors.

\section{References}

Hibbeln, J. R., Northstone, K., Evans, J., \& Golding, J. (2018). Vegetarian diets and depressive symptoms among men. Journal of Affective Disorders, 225, 1317.

Loeffler, M., Engel, C., Ahnert, P., Alfermann, D., Arelin, K., Baber, R., ... \& Thiery, J. (2015). The LIFEAdult-Study: objectives and design of a populationbased cohort study with 10,000 deeply phenotyped adults in Germany. BMC public health, 15(1), 1-14.

Matta, J., Czernichow, S., Kesse-Guyot, E., Hoertel, N., Limosin, F., Goldberg, M., ... \& Lemogne, C. (2018). Depressive symptoms and vegetarian diets: results from the constances cohort. Nutrients, 10(11), 1695.

Medawar, E., Huhn, S., Villringer, A., \& Witte, A. V. (2019). The effects of plant-based diets on the body and the brain: a systematic review. Translational psychiatry, 9(1), 1-17.

Medawar, E., Enzenbach, C., Roehr, S., Villringer, A., Riedel-Heller, S. G., \& Witte, A. V. (2020). Less animal-based food, better weight status: Associations of the restriction of animal-based product intake with body-mass-index, depressive symptoms and personality in the general population. Nutrients, 12(5), 1492. 